\title{
Not just a sponge: new functions of circular RNAs discovered
}

\author{
WANG ZeFeng \\ CAS-MPG Partner Institute of Computational Biology, Shanghai 200031, China \\ Received February 11, 2015; accepted February 12, 2015; published online February 13, 2015
}

Citation: Wang ZF. Not just a sponge: new functions of circular RNAs discovered. Sci China Life Sci, 2015, 58: 407-408, doi: 10.1007/s11427-015-4826-3

One of the most exciting findings in RNA biology is the discovery of numerous circular RNAs (circRNA) in mammalian genome. Once being considered as low abundance splicing byproducts, circRNAs are surprisingly abundant and can be generated by multiple pathways. The majority of circRNAs are generated from the RNA backsplicing in which an upstream $3^{\prime}$ splicing site (ss) is joined with a downstream $5^{\prime}$ ss. Several groups have independently demonstrated that the complementary paring of intronic sequences is sufficient to promote the biogenesis of circRNA via backsplicing. In addition, intronic circRNAs can also be generated through partial degradation of lariat RNAs that are splicing byproduct.

The vast abundance of circRNAs implied that they probably play important functions inside cells, however, the general biological roles of circRNA are still not well defined. Probably the best known example is a circRNA that contains more than 70 copies of mirRNA-7 target sites and functions as a natural microRNA sponge to antagonize mircoRNA function [1,2]. However, further analyses suggest that this microRNA sponge probably presents an extreme example rather than a common function of circRNAs. More recently, a circRNA was found to function as a specific "magnet" of RNA binding protein muscleblind [3]. Because muscleblind can function as a splicing factor, the competitive binding of muscleblind by circRNA will interfere with pre-mRNA splicing [3]. In addition, an engineered circRNA was found to function as mRNA to direct protein translation through an IRES dependent pathway [4], which blurred the divisions between coding and non-coding RNA

email: zefeng@med.unc.edu as the IRESs are poorly defined in endogenous genes. Because of the diverse sequences and cellular localization of circRNAs, these known examples may represent only a small fraction of their possible functions.

Indeed a novel function of circRNA was recently reported by Shan Ge's lab in University of Science and Technology of China, representing yet another surprise in the function of non-coding RNA [5]. The known and putative functions of circRNAs are illustrated in Figure 1. In this work, Li et al. performed a systematic identification of non-coding RNAs that are associated with RNA polymerase II using CLIP, generating more than 100 Pol II-associated circRNAs. Although these Pol II-associated circRNAs are also generated through backsplicing, most of these circRNAs contain un-spliced introns that caused these circRNA retained in the nucleus. Therefore, these circRNAs were termed as exon-intron circRNAs (or EIciRNAs). The EIciRNAs are enriched in the site of transcription, and the knocking down of EIciRNA reduced the expression of the parent mRNA [5], suggesting that these circRNAs may promote the transcription of their parent mRNAs. These observations are similar to the earlier report of circular intronic RNA [6], suggesting that the transcription activation may be a general function of circRNA containing intronic sequences. $\mathrm{Li}$ et al. further demonstrated that the EIciRNAs are also associated with U1 snRNP, which is not surprising because these circRNAs contain unspliced $5^{\prime}$ splice site in the retained intron. In addition, Li et al. provided mechanistic insight by showing that U1 snRNP is indispensable for the transcriptional activation by EIciRNAs and that the direct interaction between U1 snRNA and EIciRNA is essential for such effect.

This work provided several interesting implications of 


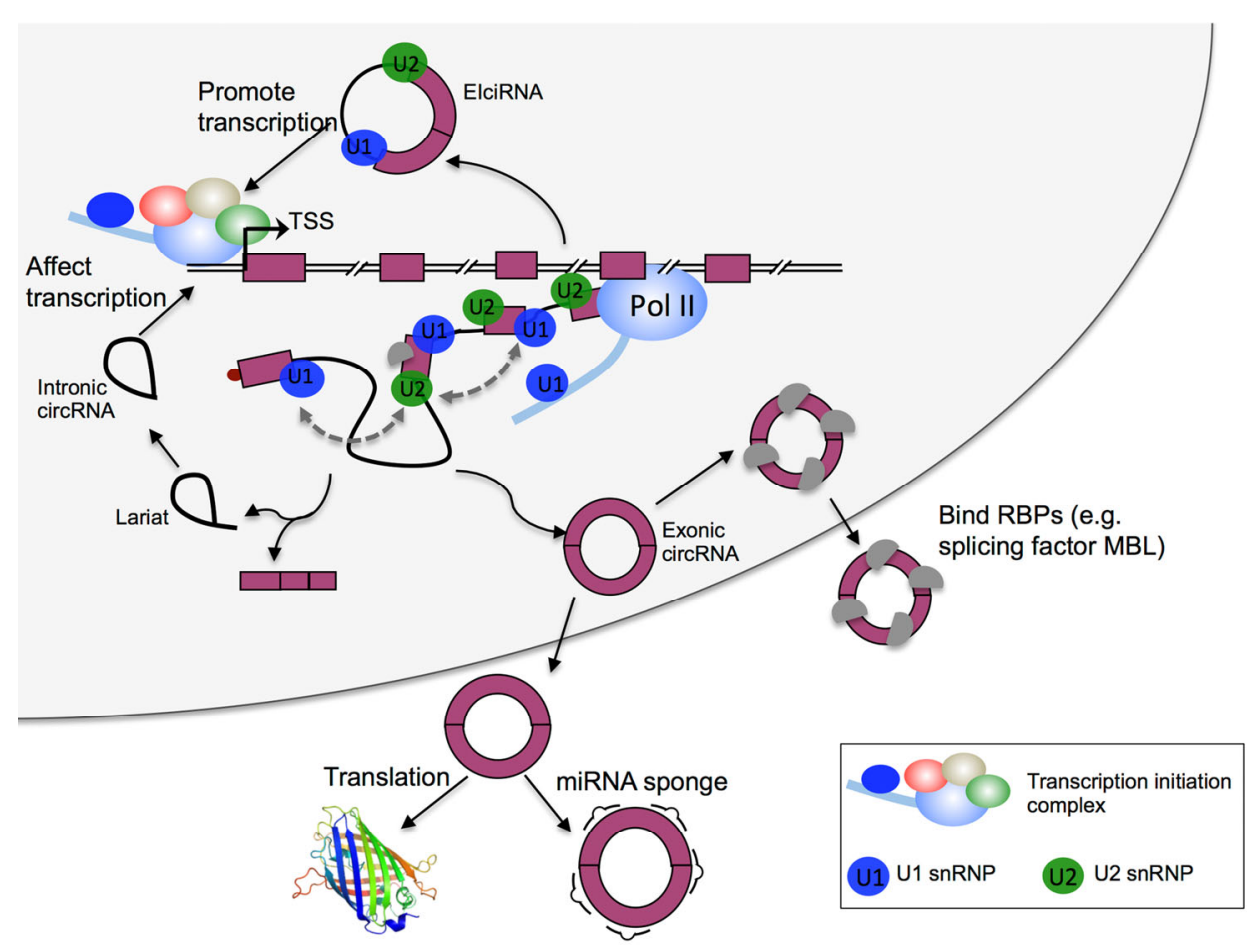

Figure 1 (color online) Diagram of common biogenesis pathway and known function of circular RNA. TSS, transcription starting site; Pol II, RNA polymerase II.

circRNA biogenesis and function. First, the identification of EIciRNA suggested that the backsplicing could happen faster than the linear splicing to generate partially spliced circRNA. This finding implies that backsplicing is a rapid process that may happen co-transcriptionally. Second, the binding of U1 snRNP by EIciRNA is essential for the positive regulation of transcription, implying that the U1 snRNP associated with other non-coding RNA (like Pol II associated long ncRNA) may also have similar function to promote transcription. Because introns and long ncRNAs contain many sequences resembling $5^{\prime}$ splice site, the recruitment of U1 snRNP to transcription site via non-coding RNAs is a highly possible scenario. Finally, it will be curious to know if the introns in EIciRNAs can be further spliced to generate exonic circRNAs that are translocated into cytoplasm. Using intronic and exonic probes, Li et al. observed that the majority of EIciRNAs are localized in the nucleus whereas the mature mRNAs are predominantly cytoplasmic. However, the existence and the localization of corresponding exonic circRNAs are still open questions.

This work also raises new questions for future investigation. For example, the mechanism of transcription activation through U1 snRNP is still unclear. Since the C-terminal domain (CTD) of Pol II can interact with U1 snRNPs to deposit them onto the elongating pre-mRNA to facilitate splicing, it is natural to speculate that the accumulation of
U1 snRNP by EIciRNA near the transcriptional site will promote this process. However, other mechanisms that are more complicated than local accumulation of U1 snRNP are also possible. Conventionally known as an essential component to catalyze pre-mRNA splicing, the U1 snRNP was later found to inhibit alternative splicing and poly-adenylation of pre-mRNA. This finding probably revealed yet another new function of U1 snRNP.

1 Memczak S, Jens M, Elefsinioti A, Torti F, Krueger J, Rybak A, Maier L, Mackowiak SD, Gregersen LH, Munschauer M, Loewer A, Ziebold U, Landthaler M, Kocks C, le Noble F, Rajewsky N. Circular RNAs are a large class of animal RNAs with regulatory potency. Nature, 2013, 495: 333-338

2 Hansen TB, Jensen TI, Clausen BH, Bramsen JB, Finsen B, Damgaard CK, Kjems J. Natural RNA circles function as efficient microRNA sponges. Nature, 2013, 495: 384-388

3 Ashwal-Fluss R, Meyer M, Pamudurti NR, Ivanov A, Bartok O, Hanan M, Evantal N, Memczak S, Rajewsky N, Kadener S. CircRNA biogenesis competes with pre-mRNA splicing. Mol Cell, 2014, 56: 55-66

4 Wang Y, Wang Z. Efficient backsplicing produces translatable circular mRNAs. RNA, 2015, 21: 172-179

5 Li ZY, Huang C, Bao C, Chen L, Lin M, Wang XL, Zhong GL, Yu B, Hu WC, Dai LM, Zhu PF, Chang ZX, Wu QF, Zhao Y, Jia Y, Xu P, Liu HJ, Shan G. Exon-Intron circular RNAs regulate transcription in the nucleus. Nat Struct Mol Biol, 2015, doi:10.1038/nsmb.2959

6 Zhang Y, Zhang XO, Chen T, Xiang JF, Yin QF, Xing YH, Zhu S, Yang L, Chen LL. Circular intronic long noncoding RNAs. Mol Cell, 2013, 51: 792-806

Open Access This article is distributed under the terms of the Creative Commons Attribution License which permits any use, distribution, and reproduction in any medium, provided the original author(s) and source are credited. 